\title{
HEISENBERG-INVARIANT KUMMER SURFACES
}

\author{
K. HULEK ${ }^{1}$, I. NIETO ${ }^{2}$ AND G. K. SANKARAN ${ }^{3}$ \\ ${ }^{1}$ Institut für Mathematik, Universität Hannover, Postfach 6009, \\ D 30060 Hannover, Germany (hulek@math.uni-hannover.de) \\ ${ }^{2}$ Cimat, A.C., Callejon de Jalisco S/N, Col. Mineral de Valenciana, \\ 36000 Guanajuato, Gto., Mexico (nieto@fractal.cimat.mx) \\ ${ }^{3}$ Department of Mathematical Sciences, University of Bath, \\ Bath BA2 7AY,UK (gks@maths.bath.ac.uk)
}

(Received 5 October 1998)

\begin{abstract}
We study, from the point of view of abelian and Kummer surfaces and their moduli, the special quintic threefold known as Nieto's quintic. It is, in some ways, analogous to the Segre cubic and the Burkhardt quartic and can be interpreted as a moduli space of certain Kummer surfaces. It contains 30 planes and has 10 singular points: we describe how some of these arise from bielliptic and product abelian surfaces and their Kummer surfaces.
\end{abstract}

Keywords: abelian surface; Kummer surface; moduli; classical algebraic geometry

AMS 1991 Mathematics subject classification: Primary 14K10; 14J25; 14J30; 14N05

\section{Preamble}

In this paper we study, from the point of view of abelian surfaces and their moduli, the special quintic threefold $N$ first described in [12] and [1], which is known as Nieto's quintic.* Nieto's quintic is, in some ways, analogous to the Segre cubic and the Burkhardt quartic and has a rich but still largely unexplored geometry. It has a double cover $\tilde{N}$ which is birationally equivalent to the moduli space $\mathcal{A}_{1,3}(2)$ of $(1,3)$-polarized abelian surfaces with a level-2 structure. $N$ contains 30 planes and has 10 singular points: we aim to understand how these arise from the abelian surfaces and their Kummer surfaces. It turns out that 15 of the planes are related to degenerate abelian surfaces, and this aspect is studied in the companion paper [5]. The other 15 planes come from a certain (reducible) Humbert surface in $\mathcal{A}_{1,3}(2)$. The 10 singular points arise similarly from another such Humbert surface. These two Humbert surfaces correspond to precisely those abelian surfaces whose minimally resolved Kummer surface is not embedded by the anti-invariant part of twice the polarization.

\footnotetext{
* This terminology is used in [7] and elsewhere and is adopted here by a majority vote of the authors.
} 


\section{Background and notation}

We need to fix some notation for the projective varieties discussed in [1] and we also need to make careful definitions concerning moduli of abelian surfaces.

$N$ is most conveniently defined as the subvariety of $\mathbb{P}^{5}$ given by

$$
\sum_{i=0}^{5} u_{i}=\sum_{i=0}^{5} u_{i}^{-1}=0,
$$

where the $u_{i}$ are homogeneous coordinates on $\mathbb{P}^{5}$. As is shown in [1], $N$ is singular at the 10 points which are equivalent to $(1: 1: 1:-1:-1:-1)$ under the permutation action of the symmetric group $\mathbb{S}_{6}$, and along the 20 lines $L_{i j k}$ given by $u_{i}=u_{j}=u_{k}=0$, which we will call desmic lines or D-lines.

$N$ contains 30 planes forming two $\mathbb{S}_{6}$-orbits of size 15 : the planes

$$
u_{\sigma(0)}+u_{\sigma(1)}=u_{\sigma(2)}+u_{\sigma(3)}=u_{\sigma(4)}+u_{\sigma(5)}=0, \quad \sigma \in \mathbb{S}_{6},
$$

which we call the S-planes; and the planes $F_{i j}$ given by

$$
u_{i}=u_{j}=0
$$

which we call the V-planes.

The double cover $\tilde{\mathbb{P}}^{5} \rightarrow \mathbb{P}^{5}$ branched along the coordinate hyperplanes induces, by pullback, a double cover $\nu: \tilde{N} \rightarrow N$, which is branched only along the V-planes.

We recall the definition of the Heisenberg group $\mathrm{H}_{2,2}$. It is a central extension

$$
0 \longrightarrow \mu_{2} \longrightarrow H_{2,2} \longrightarrow \mathbb{Z}_{2}^{\oplus 4} \longrightarrow 0,
$$

where $\mu_{2}$ is the group of square roots of unity, which acts on $V_{2,2}=\operatorname{Hom}\left(\mathbb{Z}_{2} \oplus \mathbb{Z}_{2}, \mathbb{C}\right)$ via the usual Schrödinger representation.

This induces an action of $H_{2,2}$ on $\mathbb{P}^{3}$. As explained in [1], $N$ is the closure of the locus in $\mathbb{P}^{4}=\left\{\sum u_{i}=0\right\}$ that parametrizes smooth $H_{2,2}$-invariant quartic surfaces $X \subseteq \mathbb{P}^{3}$ that contain a line. The S- and V-planes correspond to singular quartic surfaces $X$. In this paper we shall be concerned mainly with the V-planes.

We also consider the threefold $M \subseteq \operatorname{Grass}(2,4)$ which parametrizes lines in $\mathbb{P}^{3}$ contained in some Heisenberg-invariant quartic surface $X \subseteq \mathbb{P}^{3}$. In appropriate homogeneous coordinates $x_{i}$ on $\mathbb{P}^{5}$ it is given by

$$
\sum_{i=0}^{5} x_{i}^{2}=\sum_{i=0}^{5} x_{i}^{-2}=0
$$

and has an action of $H_{2,2}$, as well as a map $M \rightarrow N$ given by $u_{i}=x_{i}^{2}$.

Turning to abelian surfaces, it is necessary to be careful with definitions. What follows is all standard but is restated here for clarity. An abelian surface is a projective algebraic group of dimension 2. It is thus a projective complex torus $A$ with a group structure, including, in particular, a distinguished point $0 \in A$ and an involution $\iota: A \rightarrow A$, 
as well as a multiplication law. A polarization of type $\left(d_{1}, d_{2}\right)$ on $A$, where $d_{1}, d_{2}$ are positive integers and $d_{1} \mid d_{2}$, is an algebraic equivalence class (or cohomology class) $H \in \operatorname{NS}(A) \subseteq H^{2}(A, \mathbb{Z})$ which is the first Chern class of an ample line bundle $\mathcal{L}$ of type $\left(d_{1}, d_{2}\right)$. This makes sense even if we do not fix an origin $0 \in A$. The class $\left(1 / d_{1}\right) H$ is also an integral class and defines a polarization of type $\left(1, d_{2} / d_{1}\right)$, but $\mathcal{L}^{1 / d_{1}}$ is not well defined: if $\mathcal{M}$ is a line bundle such that $\mathcal{M}^{d_{1}} \cong \mathcal{L}$, then $\left(t_{x}^{*} \mathcal{M}^{d_{1}}\right) \cong \mathcal{L}$ also for any $d_{1}$-torsion point $x \in A$.

An abelian torsor is a principal homogeneous space for an abelian variety. By a symmetric torsor we mean a torsor $Y$ for an abelian variety $A$ on which a faithful action of Aut $A$ is also specified; in particular, there is an involution -1 on $Y$ with 16 fixed points, which one might refer to as possible origins (in that the choice of one of them as origin would make $Y$ into an abelian variety) or 2-torsion points. An example of a symmetric torsor is $Y=\operatorname{Pic}^{H} A$, for a general $H \in N S(A)$ : if $\sigma \in$ Aut $A$, then it acts on $Y$ by pullback.

The following easy fact will be useful to us.

Lemma 1.1. If $H$ is a polarization of type $(2,2 d)$, then there is a unique symmetric line bundle $\mathcal{L}_{H}$ with $c_{1}\left(\mathcal{L}_{H}\right)=H$ such that $\mathcal{L}$ is totally symmetric, that is, the square of a symmetric line bundle of type $(1, d)$.

Suppose we have an abelian variety $A$ and a symmetric line bundle $\mathcal{L}$ on $A$ such that the group $K(\mathcal{L})=\left\{\boldsymbol{x} \in A \mid t_{\boldsymbol{x}}^{*} \mathcal{L} \cong \mathcal{L}\right\}$ contains the group ${ }_{2} A$ of 2 -torsion points of $A$. As in [10] and [9], we define a theta structure of level $(2,2)$ on $A$ to be an isomorphism between the Heisenberg group $\bar{H}_{2,2}$, which is an extension

$$
0 \longrightarrow \mathbb{C}^{*} \longrightarrow \bar{H}_{2,2} \longrightarrow \mathbb{Z}_{2}^{\oplus 4} \longrightarrow 0
$$

and the group $\mathcal{G}_{2,2}(\mathcal{L})$ of theta characteristics for $\mathcal{L}$, given by

$$
0 \longrightarrow \mathbb{C}^{*} \longrightarrow \mathcal{G}_{2,2} \longrightarrow{ }_{2} A \longrightarrow 0
$$

which is the identity on $\mathbb{C}^{*}$. Such a choice induces an action of $H_{2,2}$ on $\mathcal{L}$ via the Schrödinger representation of $H_{2,2}$ and also a choice of symplectic basis for ${ }_{2} A$ over $\mathbb{Z}_{2}$ with respect to the symplectic (Weil) form induced by $H=c_{1}(\mathcal{L})$. In particular, it depends only on $c_{1}(\mathcal{L})$, not on $\mathcal{L}$ itself. The choice of symplectic basis is a level- $(2,2)$ structure in the sense of [10], often called simply a level-2 structure. We shall sometimes abuse notation by speaking of a level-2 structure for an abelian surface with a polarization of type $(1,3)$, when in fact $K(\mathcal{L})$ does not contain ${ }_{2} A$. If we do so, we mean a level-2 structure for $A$ with the polarization $c_{1}\left(\mathcal{L}^{\otimes 2}\right)$, which makes sense.

We denote by $\mathcal{A}_{1,3}(2)$ the (coarse) moduli space of abelian surfaces with a polarization of type $(1,3)$ and a level-2 structure. It makes no difference to the moduli space whether we speak of polarizations of type $(1,3)$ or $(2,6)$. We denote by $\overline{\mathcal{A}}_{1,3}(2)$ a toroidal compactification of $\mathcal{A}_{1,3}(2)$. In this paper it will not matter which toroidal compactification we take, but in [5] we are more specific.

If $(A, H, \alpha)$ is a general $(1,3)$-polarized abelian surface with a level-2 structure $\alpha$, then (cf. $[1,6.1])$ the blown-up Kummer surface $\widetilde{\mathrm{Km}} A$ has an embedding into $\mathbb{P}^{3}$ whose image is a Heisenberg-invariant quartic surface. 
Theorem 1.2. Suppose $(A, H, \alpha) \in \mathcal{A}_{1,3}(2)$. Let $\sigma: \tilde{A} \rightarrow A$ be the blow-up of $A$ in the sixteen 2-torsion points and let $f: \tilde{A} \rightarrow \widehat{\mathrm{Km}} A=\tilde{A} / \tilde{\iota}$ be the quotient by the involution on $\tilde{A}$ induced by $\iota$ on $A$. Take $\mathcal{L}=\mathcal{L}_{2 H}$, as in Lemma 1.1, and let $\tilde{\mathcal{L}}=\sigma^{*} \mathcal{L}$. Then the linear system $|\mathcal{L}|^{-}$of $\tilde{\iota}$-anti-invariant sections induces a rational map $\phi_{|\overline{\mathcal{L}}|^{-}}: \widetilde{\mathrm{Km}} A \rightarrow \mathbb{P}^{3}:$ as long as $(A, H)$ is not a product, $\phi_{|\tilde{\mathcal{L}}|^{-}}$is a morphism and the image is a quartic surface containing a line. Moreover, $\alpha$ induces an $H_{2,2}$-action on $\widetilde{\mathrm{Km}} A$ and $\tilde{\mathcal{L}}$, and $\phi_{|\tilde{\mathcal{L}}|^{-}}$is equivariant for these actions.

Proof. This is merely an assemblage of known results, restated here in a form convenient for us. The existence of the map $\phi_{|\hat{\mathcal{L}}|^{-}}$may be found in [11], [12] and [2]. In all these places it is also shown that the image is a quartic surface. The choice of a symmetric line bundle $\mathcal{M}$ such that $\mathcal{M}^{2} \cong \mathcal{L}$ determines a symmetric divisor $M_{0}$ on $A$ given by the vanishing of an anti-invariant section of $\mathcal{M}$. The image of $\sigma^{*} M_{0}$ in $\mathbb{P}^{3}$ is a line $[\mathbf{1}, \mathrm{p} .194]$, and the $H_{2,2}$-equivariance is also proved in [1].

Corollary 1.3. There is a dominant rational map $\psi: \overline{\mathcal{A}}_{1,3}(2) \rightarrow N$, which is a morphism on $\mathcal{A}_{1,3}(2)$.

Proof. The map $\psi$ is defined by $\psi(A, H, \alpha)=\phi_{|\tilde{\mathcal{L}}|^{-}}(\widetilde{\mathrm{Km}} A)$, which, by Theorem 1.2 and [1, Theorem 8.1], gives a point of $N$ as long as the 16 lines coming from the 16 choices for $\mathcal{M}$ are skew. This is true for general $A$ so $\psi$ extends to an open subset of $\overline{\mathcal{A}}_{1,3}(2)$ and also to the general point of any of the Humbert surfaces that parametrize product abelian surfaces. We shall see below (Theorem 3.2) that these Humbert surfaces are contracted to points so $\psi$ extends to every point of the Humbert surfaces and, hence, to the whole of $\mathcal{A}_{1,3}(2)$. The closure of the image of $\psi$ is an irreducible subvariety of $N$ and has dimension 3 , so $\psi$ is dominant.

In [5] we describe an extension of $\psi$ to part of the boundary.

\section{Moduli}

In this section, we shall describe the relationships between the projective varieties such as $N$ and $M$ on the one hand, and moduli spaces for abelian and Kummer surfaces on the other. We begin by restating a main result from [1].

Proposition 2.1. There is a double cover $\tilde{N}=M / H_{2,2}$ of $N$ such that $\tilde{N}$ is birationally equivalent to $\overline{\mathcal{A}}_{1,3}(2)$.

If we identify $\left(\sum x_{i}=0\right)$ with the Plücker quadric of lines in $\mathbb{P}^{3}$, then $M$ parametrizes those lines that lie on some $H_{2,2}$-invariant quartic surface $X \subseteq \mathbb{P}^{3}$. For a general $\ell \in M$ this $X$ is unique. The action of $H_{2,2}$ on $M$ is described in [1]: every element of $H_{2,2}$ changes the signs of an even number of the coordinates $x_{i}$. Of course, $-1 \in H_{2,2}$ acts trivially in projective space, so $H_{2,2}$ acts on $M$ via the quotient and the morphism $M \rightarrow \tilde{N}$ is of degree 16 . 
The squaring map $M \rightarrow N$ of degree 32 may be interpreted as the quotient map under the action of the group generated by $H_{2,2}$ and

$$
\epsilon:\left(x_{0}: x_{1}: x_{2}: x_{3}: x_{4}: x_{5}\right) \mapsto\left(-x_{0}: x_{1}: x_{2}: x_{3}: x_{4}: x_{5}\right) \text {. }
$$

It factors through $M \rightarrow \tilde{N}$, the remaining part being the double cover $\nu: \tilde{N} \rightarrow N$ given by taking the double cover $\nu: \tilde{\mathbb{P}}^{5} \rightarrow \mathbb{P}^{5}$ branched along the hyperplane sections, so $\tilde{N}=\nu^{-1}(N)$. Thus $\nu$ is induced by the squaring map: as is pointed out in [1], the spaces $\overline{\mathcal{A}}_{1,3}(2)$ and $\tilde{N}$ are birationally equivalent but the generically 2-to-1 rational map $\psi: \overline{\mathcal{A}}_{1,3}(2) \rightarrow N$ is completely different from $\nu$.

We now restate the theorem from [1] giving moduli descriptions of $M$ and $\tilde{N}$.

Theorem 2.2. $\tilde{N}$ is birationally equivalent to a compactification of the moduli space $\mathcal{A}_{1,3}(2)$ of abelian surfaces $A$ with a polarization $H$ of type $(1,3)$ and a level-2 structure $\alpha$. $M$ is birationally equivalent to a compactification of the moduli space of abelian surfaces with a symmetric bundle $\mathcal{L}$ of type $(1,3)$ and a level-2 structure.

Remark 2.3. The result proved in [1] is rather more precise, specifying open sets $M^{s}$ and $M^{s} / H_{2,2}$ in $M$ and $\tilde{N}$ and open sets in the moduli spaces where the birational equivalences are isomorphisms.

Remark 2.4. In view of Lemma 1.1, an alternative birational way of describing $\tilde{N}$ is as the moduli space of abelian surfaces with a totally symmetric line bundle $\mathcal{M}$ of type $(2,6)$ and a level-2 structure.

Remark 2.5. There is no need to speak of the moduli of $(2,6)$-polarized abelian surfaces. The version of this theorem in [7, Theorem 3.4.16, Corollary 3.4.17] is wrong, because $\mathcal{A}_{1,3}(2)$ and $\mathcal{A}_{2,6}(2)$ are the same (and so are $\Gamma_{1,3}$ and $\Gamma_{2,6}$ ); however, Hunt's discussion of the situation is scarcely affected by this slip.

Remark 2.6. There is no preferred choice of $\mathcal{L}$ : thus $M$ is a principal $\mathbb{Z}_{2}^{\oplus 4}$-bundle over $\tilde{N}$. Over a point of $M^{s} / H_{2,2}$ which is the moduli point of $(A, H, \alpha)$, the fibre may be thought of as the principal ${ }_{2} A$-space of all 16 symmetric line bundles of Chern class $H$, acted on by translation.

Strictly speaking the procedure used in [1] to prove Theorem 2.2 constructs a family of abelian torsors, not abelian surfaces, over $M^{s}$, since Nikulin's construction does not select a distinguished origin but only picks out the 16 fixed points of -1 . But this is good enough because one can replace the family by its double dual.

The moduli space $\mathcal{A}_{1,3}$ of $(1,3)$-polarized abelian surfaces is $\mathbb{H}_{2} / \Gamma_{1,3}$, where

$$
\mathbb{H}_{2}=\left\{Z=\left(\begin{array}{ll}
\tau_{1} & \tau_{2} \\
\tau_{2} & \tau_{3}
\end{array}\right) \in M_{2 \times 2}(\mathbb{C}) \mid Z={ }^{t} Z, \operatorname{Im} Z>0\right\}
$$

and $\Gamma_{1,3}$ is the paramodular group in the sense of $[3]$, that is

$$
\Gamma_{1,3}=\left\{\gamma \in \operatorname{Sp}(4, \mathbb{Q}) \mid \gamma \in\left(\begin{array}{cccc}
\mathbb{Z} & \mathbb{Z} & \mathbb{Z} & 3 \mathbb{Z} \\
3 \mathbb{Z} & \mathbb{Z} & 3 \mathbb{Z} & 3 \mathbb{Z} \\
\mathbb{Z} & \mathbb{Z} & \mathbb{Z} & 3 \mathbb{Z} \\
\mathbb{Z} & \frac{1}{3} \mathbb{Z} & \mathbb{Z} & \mathbb{Z}
\end{array}\right)\right\}
$$


$\Gamma_{1,3}$ acts on $\mathbb{H}_{2}$ by fractional linear transformations,

$$
\left(\begin{array}{ll}
A & B \\
C & D
\end{array}\right): Z \mapsto(A Z+B)(C Z+D)^{-1} .
$$

In the case of polarization of type $(1,3)$ (or type $(1, t)$ in fact) it makes sense to speak of a dual polarized variety: according to [3], one has a map $\Phi(3): \mathcal{A}_{1,3} \rightarrow \mathcal{A}_{1,3}$ that sends $(A, H)$ to $(\hat{A}, \hat{H})$, where $\hat{A}=\operatorname{Pic}^{0} A$ and $\hat{H}$ is of type $(1,3)$. It is induced [3, Proposition 1.6] by the element of order 2

$$
V_{3}=\left(\begin{array}{cccc}
0 & \sqrt{3}^{-1} & 0 & 0 \\
\sqrt{3} & 0 & 0 & 0 \\
0 & 0 & 0 & \sqrt{3} \\
0 & 0 & \sqrt{3}^{-1} & 0
\end{array}\right) \in \mathrm{Sp}(4, \mathbb{R})
$$

also acting on $\mathbb{H}_{2}$ by a fractional linear transformation.

$\mathcal{A}_{1,3}(2)$ is the quotient $\mathbb{H}_{2} / \Gamma_{1,3}(2)$, where $\Gamma_{1,3}(2)<\mathrm{Sp}(4, \mathbb{Q})$ is given by

$$
\Gamma_{1,3}(2)=\left\{\gamma \in \operatorname{Sp}(4, \mathbb{Q}) \mid \gamma-I \in\left(\begin{array}{cccc}
2 \mathbb{Z} & 2 \mathbb{Z} & 2 \mathbb{Z} & 6 \mathbb{Z} \\
6 \mathbb{Z} & 2 \mathbb{Z} & 6 \mathbb{Z} & 6 \mathbb{Z} \\
2 \mathbb{Z} & 2 \mathbb{Z} & 2 \mathbb{Z} & 6 \mathbb{Z} \\
2 \mathbb{Z} & \frac{2}{3} \mathbb{Z} & 2 \mathbb{Z} & 2 \mathbb{Z}
\end{array}\right)\right\} .
$$

$V_{3}^{-1} \Gamma_{1,3}(2) V_{3}=\Gamma_{1,3}(2)$, so the involution $\Phi(3)$ induces an involution on $\mathcal{A}_{1,3}(2)$ with the same properties: we shall call this $\Phi(3)$ also.

There is an alternative moduli description of $\tilde{N}$ as a space of torsors. Because we start with an $\mathrm{H}_{2,2}$ orbit of lines, rather than a particular one, this is what the geometry really produces.

Proposition 2.7. $\tilde{N}$ is birationally equivalent to a compactification of the moduli space of symmetric abelian torsors $Y$ of dimension 2 with a symmetric bundle $\mathcal{L}$ of type $(1,3)$ and a level-2 structure $\alpha$.

Proof. Given a triple $(A, H, \alpha) \in \mathcal{A}_{1,3}(2)$, where $A$ is an abelian variety, we associate to it $(\hat{A}, \mathcal{L}, \hat{\alpha})$, where $\hat{A}$ is the torus obtained by forgetting the origin of $A$, and $\mathcal{L}$ is a symmetric line bundle representing $H$. If we choose another symmetric line bundle $\mathcal{L}^{\prime}$ with $c_{1}\left(\mathcal{L}^{\prime}\right)=H$, then there is a 2-torsion point $x$ with $t_{x}^{*} \mathcal{L}=\mathcal{L}^{\prime}$. This translation defines an automorphism of the torsor and, hence, this map is well defined up to isomorphism. The level-2 structure $\hat{\alpha}$ is simply a level-2 structure on the underlying abelian surface. Inversely, starting with $(Y, \mathcal{L}, \hat{\alpha})$ with $Y$ a symmetric torsor, we make $Y$ into an abelian surface $A$ by choosing one of the 16 fixed points of the involution -1 , say $\boldsymbol{x}$, as the origin. We take $H=c_{1}(\mathcal{L})$ and $\alpha=\hat{\alpha}$. We must check that this definition does not depend on the choice of $\boldsymbol{x}$. If we choose a different 2-torsion point $\boldsymbol{x}^{\prime}$ instead of $\boldsymbol{x}$, we get an abelian surface $A^{\prime}$, and the translation $t_{x^{\prime}-x}$ defines an isomorphism from $A$ to $A^{\prime}$. It maps $\mathcal{L}$ to a different line bundle $\mathcal{L}^{\prime}$, but $c_{1}(\mathcal{L})=c_{1}\left(\mathcal{L}^{\prime}\right)$, so, again at the level of isomorphism classes, the map is well defined; and the two maps are obviously inverse to each other. 
The above argument works for any polarization (and indeed any dimension). In our case it is also possible to use $\Phi(3)$ and the totally symmetric bundle of class $2 H$ to induce an isomorphism.

A symmetric bundle of type $(1,3)$ even determines a unique divisor on $Y$, because the space of anti-invariant sections under the action of -1 is 1-dimensional. So $Y$ is very like a Jacobian but the 'theta divisor' gives a non-principal polarization.

The relevance of $\Phi(3)$ is that it gives rise to $\psi$.

Proposition 2.8. There exists $g \in \Gamma_{1,3} / \Gamma_{1,3}(2)$ such that if $x_{1}, x_{2} \in \mathcal{A}_{1,3}(2)$ and $\psi\left(x_{1}\right)=\psi\left(x_{2}\right)$, then $\Phi(3)\left(g x_{1}\right)=x_{2}$.

Proof. Suppose $x_{i}=\left(A_{i}, H_{i}, \alpha_{i}\right)$ are such that $\psi\left(x_{1}\right)=\psi\left(x_{2}\right)$. For general $x_{i}$ we know that $\mathrm{Km} A_{1} \cong \mathrm{Km} A_{2}$, since $\phi_{|\tilde{\mathcal{L}}|}$ is an isomorphism onto its image. By [3, Theorem 1.5], we also know that, for general $A_{i}$, if $\mathrm{Km} A_{1} \cong \mathrm{Km} A_{2}$, then $\left(A_{2}, H_{2}\right)=\left(\hat{A}_{1}, \hat{H}_{1}\right)$. So the map $\mathcal{A}_{1,3}(2) \rightarrow \mathcal{A}_{1,3}(2)$ induced by $\psi$ agrees with $V_{3}$ up to a possible change of the level structure, that is, up to the action of $\Gamma_{1,3} / \Gamma_{1,3}(2)$.

The element $g V_{3}$ is an involution modulo $\Gamma_{1,3}(2)$. There are, in principle, two possibilities. One is that $g V_{3}$ itself is an involution. (This happens if $g=1$, but also for some other g.) By [3, Corollary 3.9], there is exactly one involution in $\Gamma_{1,3} V_{3}$ up to conjugation with $\Gamma_{1,3}$. The fixed locus of such an involution is an Humbert surface of discriminant 12 in $\mathcal{A}_{1,3}(2)$. For a general element of this Humbert surface the linear system $\phi_{|\tilde{\mathcal{L}}|^{-}}$is an embedding. But then this would contradict Statement 8.4 in [1], which says that $\psi$ is unbranched on $M^{s} / H_{2,2}$. In particular, we see that $g \neq 1$. The other possibility is that $\left(g V_{3}\right)^{2} \in \Gamma_{1,3}(2)$, but $\left(g V_{3}\right)^{2} \neq 1$. It follows, again by [3, Corollary 3.9], that the map $\psi$ is not branched along an Humbert surface of discriminant 4. On the other hand, the branch locus of the map $\nu$ is the image of the locus of bielliptic abelian surfaces (see below). These surfaces are parametrized by an Humbert surface of discriminant 4 . Hence we can see directly from moduli that the two maps $\psi$ and $\nu$ do not agree.

\section{Branching and special abelian surfaces}

In this section we study the branch locus of $\nu$ below $\mathcal{A}_{1,3}(2)$ and the corresponding abelian surfaces. Recall that we define $\psi: \mathcal{A}_{1,3}(2) \rightarrow\left|\mathcal{O}_{\beth 3}(4)\right|$ by

$$
\psi(A, H, \alpha)=X=\operatorname{Im} \phi_{|\bar{c}|^{-}} \subseteq \mathbb{P}^{3}
$$

By continuity, $\psi(A, H, \alpha) \in N$, even if $X$ is singular.

The image of the map $\phi_{|\overline{\mathcal{L}}|^{-}}$was studied by Bauer [2]. There is a classical study of quartic surfaces in $\mathbb{P}^{3}$ with singularities by Jessop [8]. We begin, though, with the double points of $N$. These are in the S-planes, but, unlike other points in the S-planes, they can arise from smooth abelian surfaces. According to the main theorem of [2], the map $\phi_{|\tilde{\mathcal{L}}|^{-}}: \widetilde{\mathrm{Km}} A \rightarrow \mathbb{P}^{3}$ is usually an isomorphism onto its image (this is also shown in [1] and [11]), but there are exceptional cases, numbered (III), (IV) and (V) in $[2, \S 5]$. 
(III) The (1,3)-polarization $H$ is given by $\mathcal{O}_{A}(G+E)$ with $E$ elliptic and $G$ an irreducible genus 2 curve with $G \cdot E=2$. In this case, $\phi_{|\overline{\mathcal{L}}|^{-}}$is birational and the image $X$ has four nodes.

(IV) $H$ is given by $\mathcal{O}_{A}\left(E_{1}+E_{2}+E_{3}\right), E_{i}$ elliptic, $E_{i} \cdot E_{j}=1-\delta_{i j}$. This is a degenerate case of (III) and the image $X$ has twelve nodes. $\phi_{|\tilde{\mathcal{L}}|}$ is still birational.

(V) $A=E_{1} \times E_{2}$ and $H=c_{1}\left(\mathcal{O}_{A}\left(E_{1}+3 E_{2}\right)\right), E_{i}$ elliptic. The image $X$ is smooth but $\phi_{|\tilde{\mathcal{L}}|^{-}}$is 2-to-1.

Lemma 3.1. If a group $G$ acts transitively on a set $X \ni x_{0}$ and $G^{\prime}$ is a normal subgroup of $G$ of finite index, and if $S$ and $S^{\prime}$ are the stabilizers of $x_{0}$ in $G$ and $G^{\prime}$, respectively, then the number of $G^{\prime}$-orbits in $X$ is equal to $\left[G: G^{\prime}\right] /\left[S: S^{\prime}\right]$.

Proof. This is an elementary calculation (cf. [4, Lemma 2.2]).

Theorem 3.2. The nodes in $N$ correspond to points of $\mathcal{A}_{1,3}(2)$ where $A=E_{1} \times E_{2}$ and $H=c_{1}\left(\mathcal{O}_{A}\left(E_{1}+3 E_{2}\right)\right)$. These points form a surface with 20 irreducible components, which are contracted by $\mathcal{A}_{1,3}(2) \rightarrow N$ to the 10 nodes of $N$.

Proof. The 10 nodes of $N$ are the $\mathbb{S}_{6}$-translates of $(1: 1: 1:-1:-1:-1) \in \mathbb{P}^{4}$ and they correspond to non-reduced quartic surfaces, namely the squares of the fundamental quadrics. This is checked for the point $(-1: 1: 1: 1:-1:-1)$ (which is enough) in $[1$, p. 190]. As there are no other Heisenberg-invariant quadrics, any non-reduced $H_{2,2}$-invariant quartic in $\mathbb{P}^{4}$ is the square of a fundamental quadric and corresponds to a node of $N$.

Suppose $A=E_{1} \times E_{2}$ and $H=c_{1}\left(\mathcal{O}_{E_{1}}(3) \otimes \mathcal{O}_{E_{2}}(1)\right)$ is a product polarization of type $(1,3)$ on $A$. Then we are in case (V) of [2] and, therefore, by the main theorem of [2], the rational map $\phi_{|\tilde{\mathcal{L}}|^{-}}: \tilde{A} \rightarrow \mathbb{P}^{3}$ is not defined on certain base curves of $|\tilde{\mathcal{L}}|^{-}$, namely the symmetric translates of $E_{2}$. However, away from these base curves, $\phi_{|\tilde{\mathcal{L}}|^{-}}$coincides with the morphism $\tilde{A} \rightarrow \mathbb{P}^{3}$ coming from the polarization $c_{1}\left(\mathcal{O}_{E_{1}}(2) \otimes \mathcal{O}_{E_{2}}(2)\right)$ of type $(2,2)$, which is of degree 2. So the closure of the image of $\phi_{|\tilde{c}|^{-}}$in $\mathbb{P}^{3}$ is a (double) quadric: if we make a choice of level-2 structure so as to fix an $\mathrm{H}_{2,2}$-action, the image will then be $H_{2,2}$-invariant so it must be one of the 10 fundamental quadrics. So the image of the locus

$$
\left\{(A, H, \alpha) \in \mathcal{A}_{1,3}(2) \mid(A, H) \cong\left(E_{1} \times E_{2}, c_{1}\left(\mathcal{O}_{E_{1}}(3) \otimes \mathcal{O}_{E_{2}}(1)\right)\right)\right\}
$$

of product surfaces with product polarization, in $N$ is contained in the 10 nodes. Since the nodes are permuted transitively by the action of $\mathbb{S}_{6}$, the image must be all the nodes.

Conversely, if $\psi(A, H, \alpha)$ is one of the nodes, then $(A, H, \alpha)$ must be a product since (by [2]) in other cases the map $\phi_{|\tilde{L}|^{-}}: \widetilde{\mathrm{Km}}(A) \rightarrow \mathbb{P}^{3}$ is birational onto its image, which is therefore irreducible and birationally a K3 surface.

To prove the rest of the theorem, we need to count the number of irreducible components of the space

$$
\{(A, H, \alpha) \mid(A, H) \text { a product, } H \text { of type }(1,3), \alpha \text { a level-2 structure }\}
$$


in $\mathcal{A}_{1,3}(2)$. We use the same method as [4], making use of the description of $\mathcal{A}_{1,3}(2)$ as a Siegel modular variety.

Consider the surface $\hat{\mathcal{H}}_{1}=\left\{Z \in \mathbb{H}_{2} \mid \tau_{2}=0\right\}$ and its image $\mathcal{H}_{1}$ in $\mathcal{A}_{1,3}$. It is shown in [4] that $\mathcal{H}_{1}$ parametrizes abelian surfaces of type $(1,3)$ which split as polarized abelian surfaces, that is, product surfaces with the product polarization. $\mathcal{H}_{1}$ is irreducible, because $\hat{\mathcal{H}}_{1}$ is. We want to know the number of components of $\pi^{*}\left(\mathcal{H}_{1}\right)$, where $\pi: \mathcal{A}_{1,3}(2) \rightarrow \mathcal{A}_{1,3}$ is the quotient map by the action of $\Gamma_{1,3} / \Gamma_{1,3}(2) \cong \mathrm{Sp}\left(4, \mathbb{Z}_{2}\right)$ (which is $\mathbb{S}_{6}$ as an abstract group).

We look at the stabilizers

$$
S_{1}=\left\{\gamma \in \Gamma_{1,3} \mid \gamma\left(\hat{\mathcal{H}}_{1}\right)=\hat{\mathcal{H}}_{1}\right\} \quad \text { and } \quad S_{1}(2)=\left\{\gamma \in \Gamma_{1,3}(2) \mid \gamma\left(\hat{\mathcal{H}}_{1}\right)=\hat{\mathcal{H}}_{1}\right\}
$$

As in [4] one has

$$
S_{1}=\left\{\left(\begin{array}{cccc}
a & 0 & b & 0 \\
0 & a^{\prime} & 0 & b^{\prime} \\
c & 0 & d & 0 \\
0 & c^{\prime} & 0 & d^{\prime}
\end{array}\right) \mid\left(\begin{array}{cc}
a & b \\
c & d
\end{array}\right),\left(\begin{array}{cc}
a^{\prime} & b^{\prime} \\
c^{\prime} & d^{\prime}
\end{array}\right) \in \mathrm{SL}(2, \mathbb{Z})\right\}
$$

so $S_{1} \cong \mathrm{SL}(2, \mathbb{Z}) \times \mathrm{SL}(2, \mathbb{Z})$, and an element of $S_{1}$ is in $\Gamma_{1,3}(2)$, and hence in $S_{1}(2)$, if and only if

$$
\left(\begin{array}{ll}
a & b \\
c & d
\end{array}\right) \text { and }\left(\begin{array}{ll}
a^{\prime} & b^{\prime} \\
c^{\prime} & d^{\prime}
\end{array}\right)
$$

are both congruent to the identity $\bmod 2$. So $S_{1} / S_{1}(2) \cong \operatorname{SL}\left(2, \mathbb{Z}_{2}\right) \times \operatorname{SL}\left(2, \mathbb{Z}_{2}\right)$.

Now we can conclude the proof of Theorem 3.2 by applying Lemma 3.1 . We take $G=\Gamma_{1,3}, G^{\prime}=\Gamma_{1,3}(2)$, and $X=\left\{\gamma \hat{\mathcal{H}}_{1} \mid \gamma \in \Gamma_{1,3}\right\}$, the set of $\Gamma_{1,3}$-translates of $\hat{\mathcal{H}}_{1}$ or of preimages of $\mathcal{H}_{1}$ in $\mathbb{H}_{2}$. The set $X / G^{\prime}$ is precisely the set of preimages of $\mathcal{H}_{1}$ in $\mathcal{A}_{1,3}(2)$, and, by Lemma 3.1 , it has

$$
\frac{\left[\Gamma_{1,3}: \Gamma_{1,3}(2)\right]}{\left[S_{1}: S_{1}(2)\right]}=\frac{\left|\mathrm{Sp}\left(4, \mathbb{Z}_{2}\right)\right|}{\left|\mathrm{SL}\left(2, \mathbb{Z}_{2}\right) \times \mathrm{SL}(2, \mathbb{Z})\right|}=\frac{720}{36}=20
$$

elements. This is, therefore, the number of irreducible surfaces in $\mathcal{A}_{1,3}(2)$ corresponding to product surfaces.

The nodes lie in the S-planes in $N$. We have said that, in general, points in the S-planes will correspond to degenerate abelian surfaces, but here the closure of an Humbert surface in $\overline{\mathcal{A}}_{1,3}(2)$ is contracted by $\psi$ to a point that we may also think of as coming from the boundary $\overline{\mathcal{A}}_{1,3}(2) \backslash \mathcal{A}_{1,3}(2)$. Of course, other components in the boundary may also be contracted to the nodes (this will depend on our choice of toroidal compactification), but only the nodes come from both degenerate and non-degenerate abelian surfaces.

Theorem 3.3. If $\boldsymbol{u} \in N$ is a point in some $S$-plane and there is an abelian surface $(A, H, \alpha) \in \mathcal{A}_{1,3}(2)$ such that $\psi(A, H, \alpha)=u$, then $u$ is a node and $(A, H, \alpha)$ is a product. 
Proof. If $(A, H, \alpha)$ is not a product, then $\phi_{|\tilde{\mathcal{L}}|^{-}}: \widetilde{\mathrm{Km}}(A) \cdots X$ is birational onto its image, which is, therefore, an irreducible variety which is birationally a K3 surface. But for any $\boldsymbol{u}$ on an S-plane the corresponding quartic surface contains a double line (actually a pair of double lines). Following [8, ch. VI, §77], we observe that a plane containing this line cuts out a conic on $X$. So $X$ is covered by a family of rational curves and is, therefore, ruled.

Now we turn to the V-planes $F_{i j}$, defined by $u_{i}=u_{j}=0$, which form the branch locus of $\nu: \tilde{N} \rightarrow N$. There are 15 of these planes, equivalent under the $\mathbb{S}_{6}$-action on $\mathbb{P}^{5}$ : we may choose one of them to work with and we use $F_{45} . F_{45}$ determines the family

$$
\mathcal{F}_{A E}=\left\{B\left(z_{0}^{2} z_{1}^{2}+z_{2}^{2} z_{3}^{2}\right)+C\left(z_{0}^{2} z_{2}^{2}+z_{1}^{2} z_{3}^{2}\right)+D\left(z_{0}^{2} z_{3}^{2}+z_{1}^{2} z_{2}^{2}\right)=0\right\}
$$

which is studied in $[\mathbf{1 3}]$.

Theorem 3.4. Let $u \in N$ be a point of a $V$-plane, not lying on an $S$-plane nor on one of the $D$-lines $\left\{u_{i}=u_{j}=u_{k}=0\right\}$. Then there exists an abelian variety $A$ with a polarization $H$ of type $(1,3)$ and a level-2 structure $\alpha$, such that $\psi(A, H, \alpha)=\boldsymbol{u}$ and $(A, H)$ is a bielliptic abelian surface in the sense of [4]. In particular, $A$ is isomorphic to $E_{1} \times E_{2} / \mathbb{Z}_{2} \times \mathbb{Z}_{2}$ for some elliptic curves $E_{1}, E_{2}$ and some action of $\mathbb{Z}_{2} \times \mathbb{Z}_{2}$.

Proof. The conditions on $\boldsymbol{u}$ imply that the corresponding quartic surface $X_{\boldsymbol{u}}=X$ (given by $B=\frac{1}{2}\left(-u_{0}-u_{1}+u_{2}+u_{3}\right)$, etc.; see $[\mathbf{1}, \mathbf{1 3}]$ ) has four simple nodes and no other singularities. As in [13], we can use the theorem of Nikulin [14] to construct a diagram

$$
A \stackrel{\sigma}{\leftarrow} \tilde{A} \stackrel{f}{\rightarrow} \tilde{X} \stackrel{\beta}{\rightarrow} X,
$$

where $\beta$ is the blow-up of the four nodes of $X$ and $f: \tilde{A} \rightarrow \tilde{X}$ is the double cover branched along a set $L_{0}$ of 16 disjoint smooth rational curves. $L_{0}$ is the pullback to $\tilde{X}$ of one of the two $H_{2,2}$-orbits of lines in $X$, which become disjoint after the blow-up $\beta$. We consider the curves $E_{0}=\sigma_{*} f^{*}\left(\beta^{-1}\left(p_{0}\right)\right)$, where $p_{0}$ is one of the nodes, and $\Theta=\sigma_{*} f^{*} \beta^{*} L^{\prime}$, where $L^{\prime}$ is another line on $X$. Then (see [13, Lemma 6-3]) $g\left(E_{0}\right)=1$ and $g(\Theta)=2$.

For reasons explained below, we now diverge from the line of argument given in [13]. We have $E_{0} \cdot \theta=2$ and there is an exact sequence

$$
0 \longrightarrow E_{0} \longrightarrow A \longrightarrow E_{0}^{\prime} \longrightarrow 0
$$

for some elliptic curve $E_{0}^{\prime}$. So the map $\pi: A \rightarrow E_{0}^{\prime}$ induces a double cover $\Theta \rightarrow E_{0}^{\prime}$. By Torelli, $\left(A, \mathcal{O}_{A}(\Theta)\right)=(\operatorname{Jac} \Theta, \Theta)$, so $A$ is the Jacobian of a bielliptic genus 2 curve, i.e. a principally polarized bielliptic abelian surface. By [4, Proposition 4.1], it follows that $A=E_{1} \times E_{2} / \mathbb{Z}_{2} \times \mathbb{Z}_{2}$ for suitable elliptic curves $E_{1}, E_{2}$. The polarization that we are really interested in, however, is $H=E_{0}+\Theta$, which is of type $(1,3)$. The bielliptic involution $j: \theta \rightarrow \Theta$ induces $j_{A}:(A, \Theta) \rightarrow(A, \theta)$ and $\pi: A \rightarrow E_{0}^{\prime}$ factors through $j_{A}$. Therefore $j_{A}$ preserves $E_{0}$, which is a fibre of $\pi$, so $j_{A}$ induces an involution of $(A, H)$ as well. Hence (cf. [4]) $(A, H)$ either splits as a polarized abelian surface or else is a bielliptic $(1,3)$-polarized abelian surface. The first case can be excluded, because we already know 
that the linear system $|2 H|^{-}$induces a map of $\widetilde{\mathrm{Km}} A$ which is birational onto its image. (Looking carefully at the proof of [4, Proposition 4.4], one can show that every bielliptic $(1,3)$-polarized abelian surface contains a bielliptic genus 2 curve, and conversely.) The level-2 structure on $(A, H)$ is simply induced by the $H_{2,2}$-action on $X$.

Remark 3.5. In [13], the second elliptic curve and the $\mathbb{Z}_{2} \times \mathbb{Z}_{2}$-action are constructed more directly, but under the additional assumption that $\rho=\operatorname{rk} \mathrm{NS}(A)=2$. Also, there is a gap in the proof of $[\mathbf{1 3}$, Lemma 6-3(2)]. Nevertheless, we could have used this method. The restriction $\rho=2$ is harmless to us because it is true for almost all bielliptic surfaces, and the gap can easily be filled. We take the opportunity to do this.

In $\mathrm{NS}(A)$ we have (see [13, p. 333]) inequivalent elliptic curves $E, E^{\prime}$ and a genus 2 curve $\Theta^{\prime}$ with $E \cdot \Theta^{\prime}=2$. So in $\mathrm{NS}(A) \otimes \mathbb{Q}$ we have $E^{\prime}=n_{0} E+n_{1} \Theta^{\prime}$ and therefore $n_{1}=-2 n_{0} \neq 0$. It does not follow at once, as asserted in [13], that $n_{0}= \pm 1$, because we do not know that $n_{0} \in \mathbb{Z}$. The elliptic curves, however, are not divisible in $\mathrm{NS}(A)$, because, topologically, $A=E \times T$ for some real subtorus $T \subset A$. So the denominator of $n_{0}$ is at most 2 , otherwise $E^{\prime}$ is divisible, and if $n_{0} \in \mathbb{Z}$ then $n_{0}= \pm 1$. So suppose $n_{0}=k+\frac{1}{2}$. Then $2 E^{\prime}=(2 k+1) E-(4 k+2) \Theta^{\prime}$, so $E=2\left(E^{\prime}+(2 k+1) \Theta^{\prime}-k E\right)$ is divisible. So $n_{0}$ and $n_{1}$ are in fact integers and the argument in $[13]$ can be used.

Corollary 3.6. The closures of the following three loci in $\overline{\mathcal{A}}_{1,3}(2)$ coincide:

(i) the locus of $(A, H, \alpha)$ such that $(A, H)$ is a $(1,3)$-polarized bielliptic abelian surface;

(ii) the locus of $(A, H, \alpha)$ such that $\phi_{|\tilde{\mathcal{L}}|^{-}}(\widetilde{\mathrm{Km}} A)=X_{\boldsymbol{u}}$ for some $u$ in a V-plane, not in an S-plane or on a $D$-line;

(iii) the locus of $(A, H, \alpha)$ such that $H=c_{1}\left(\mathcal{O}_{A}(E+\theta)\right)$ with $E \cdot \theta=2$ and $\Theta \subseteq A$ a symmetric irreducible curve of genus 2 .

Moreover, the loci in (ii) and (iii) coincide precisely.

Proof. We have shown that (ii) implies (i) in Theorem 3.4. By the main theorem of [2], the hypotheses of (iii) imply that $|\tilde{\mathcal{L}}|^{-}$contracts the images in $\widetilde{\mathrm{Km}} A$ of the four symmetric translates of $E$ and no more, so that $\phi_{|\tilde{\mathcal{L}}|-}(\widetilde{\mathrm{Km}} A)$ is an $H_{2,2}$-invariant quartic surface with four nodes. These are precisely those parametrized by the points of the V-planes with the exceptions given in (ii), so (ii) and (iii) are the same. Finally, the locus of bielliptic surfaces without level structure in $\mathcal{A}_{1,3}$ and the locus of Jacobians of bielliptic genus 2 curves in the moduli space of principally polarized abelian surfaces are both irreducible surfaces, as is shown in [4]. The locus of such Jacobians can also be thought of as a subvariety of $\mathcal{A}_{1,3}$, by replacing the principal polarization $\theta$ by $E_{0}+\Theta$ as above: there is a unique way to do this. The two irreducible surfaces in $\mathcal{A}_{1,3}$ must therefore coincide, so the closures of the loci in (i) and (iii) are the same. In fact, the loci in (i) and (iii) coincide precisely if we drop the irreducibility condition from (iii), as one can deduce from the proof of [4, Proposition 1.4]. 
Now we know that the $\mathrm{V}$-planes in $N$ are precisely the image under $\psi$ of the surface in $\mathcal{A}_{1,3}(2)$ parametrizing bielliptic surfaces of type $(1,3)$; that is, of $\pi^{-1}\left(\mathcal{H}_{2}\right)$, where $\pi: \mathcal{A}_{1,3}(2) \rightarrow \mathcal{A}_{1,3}$ is the quotient map by $\operatorname{Sp}\left(4, \mathbb{Z}_{2}\right)$ as before and $\mathcal{H}_{2} \subseteq \mathcal{A}_{1,3}$ parametrizes bielliptic abelian surfaces. We can count the irreducible components of $\pi^{-1}\left(\mathcal{H}_{2}\right)$ just as we did for $\pi^{-1}\left(\mathcal{H}_{1}\right)$.

Theorem 3.7. The surface in $\mathcal{A}_{1,3}(2)$ parametrizing triples $(A, H, \alpha)$ for which $(A, H)$ is bielliptic has 15 irreducible components.

Proof. We put $\hat{\mathcal{H}}_{2}=\left\{Z \in \mathbb{H}_{2} \mid 3 \tau_{1}=2 \tau_{2}\right\}$ as in [4]. We know, also from [4], that the image of $\hat{\mathcal{H}}_{2}$ in $\mathcal{A}_{1,3}$ is $\mathcal{H}_{2}$, so, according to Lemma 3.1 and using the same reasoning as in Theorem 3.2, the number of components of $\pi^{-1}\left(\mathcal{H}_{2}\right)$ is

$$
\frac{\left[\Gamma_{1,3}: \Gamma_{1,3}(2)\right]}{\left[S_{2}: S_{2}^{\prime}\right]}=\frac{720}{\left[S_{2}: S_{2}(2)\right]},
$$

where $S_{2}$ and $S_{2}^{\prime}$ are the stabilizers of $\hat{\mathcal{H}}_{2}$ in $\Gamma_{1,3}$ and $\Gamma_{1,3}(2)$, respectively. From [4] we have

$$
\begin{aligned}
& S_{2}=\left\{\begin{array}{cccc}
a & 0 & 2 b & 3 b \\
\frac{3}{2}\left(a-a^{\prime}\right) & a^{\prime} & 3 b & \frac{3}{2}\left(3 b+b^{\prime}\right) \\
\frac{1}{2}\left(3 c^{\prime}+c\right) & -c^{\prime} & d & \frac{3}{2}\left(d-d^{\prime}\right) \\
-c^{\prime} & \frac{2}{3} c^{\prime} & 0 & d^{\prime}
\end{array}\right) \\
& \left.\mid M=\left(\begin{array}{ll}
a & b \\
c & d
\end{array}\right), M^{\prime}=\left(\begin{array}{ll}
a^{\prime} & b^{\prime} \\
c^{\prime} & d^{\prime}
\end{array}\right) \in \mathrm{SL}(2, \mathbb{Z}), M \equiv M^{\prime} \bmod 2\right\} \\
& \cong\left\{\left(M, M^{\prime}\right) \in \mathrm{SL}(2, \mathbb{Z}) \times \mathrm{SL}(2, \mathbb{Z}) \mid M \equiv M^{\prime} \bmod 2\right\} .
\end{aligned}
$$

Such a matrix will be in $\Gamma_{1,3}(2)$ if and only if $M \equiv M^{\prime} \equiv I \bmod 2$ and $M \equiv M^{\prime} \bmod 4$, because we need $a, a^{\prime}, d, d^{\prime}$ odd, $b, c^{\prime}$ even (so $b^{\prime}$ and $c$ also even), and $\frac{3}{2}\left(a-a^{\prime}\right), \frac{3}{2}\left(3 b+b^{\prime}\right)$, $\frac{1}{2}\left(3 c^{\prime}+c\right)$ and $\frac{3}{2}\left(d-d^{\prime}\right)$ all even.

Now consider the tower of groups

$$
\begin{aligned}
& \tilde{S}_{2}=\left\{\left(M, M^{\prime}\right) \mid M \equiv M^{\prime} \bmod 2\right\} \\
& \tilde{S}_{2}^{\prime \prime}=\left\{\left(M, M^{\prime}\right) \mid M \equiv M^{\prime} \equiv I \bmod 2\right\} \\
& \tilde{S}_{2}^{\prime}=\left\{\left(M, M^{\prime}\right) \mid M \equiv M^{\prime} \equiv I \bmod 2, M \equiv M^{\prime} \bmod 4\right\} .
\end{aligned}
$$

Then the map

$$
\left(M, M^{\prime}\right) \mapsto\left(\begin{array}{cccc}
a & 0 & 2 b & 3 b \\
\frac{3}{2}\left(a-a^{\prime}\right) & a^{\prime} & 3 b & \frac{3}{2}\left(3 b+b^{\prime}\right) \\
\frac{1}{2}\left(3 c^{\prime}+c\right) & -c^{\prime} & d & \frac{3}{2}\left(d-d^{\prime}\right) \\
-c^{\prime} & \frac{2}{3} c^{\prime} & 0 & d^{\prime}
\end{array}\right)
$$


induces isomorphisms between $S_{2}$ and $\tilde{S}_{2}$ and $S_{2}^{\prime}$ and $\tilde{S}_{2}^{\prime}$, so $\left[S_{2}: S_{2}^{\prime}\right]=\left[\tilde{S}_{2}: \tilde{S}_{2}^{\prime}\right]$. Now $\tilde{S}_{2}^{\prime \prime}$ is a normal subgroup of $\tilde{S}_{2}$ and the quotient is isomorphic to $\operatorname{SL}\left(2, \mathbb{Z}_{2}\right)$, of order 6 , and the cosets of $\tilde{S}_{2}^{\prime}$ in $\tilde{S}_{2}^{\prime \prime}$ are determined by the residue class of $M \bmod 4$. There are eight of these: the off-diagonal elements may be 0 or $2 \bmod 4$, and the diagonal ones are either both 1 or both -1 . So $\left[\tilde{S}_{2}^{\prime \prime}: \tilde{S}_{2}^{\prime}\right]=8$, so $\left[\tilde{S}_{2}: \tilde{S}_{2}^{\prime}\right]=\left[\tilde{S}_{2}: \tilde{S}_{2}^{\prime \prime}\right]\left[\tilde{S}_{2}^{\prime \prime}: \tilde{S}_{2}^{\prime}\right]=48$. So the number of irreducible components is $720 / 48=15$.

Finally, we consider the D-lines $L_{i j k}=\left\{u_{i}=u_{j}=u_{k}=0\right\} \subseteq N$ and examine the abelian surfaces that live over them. We work with the line $L_{045}$. On this line, the quintic surface $X$ has the equation

$$
C\left(z_{0}^{2}-z_{3}^{2}\right)\left(z_{1}^{2}-z_{2}^{2}\right)+D\left(z_{0}^{2}-z_{2}^{2}\right)\left(z_{3}^{2}-z_{1}^{2}\right)=0 .
$$

These are the surfaces known as desmic* surfaces in [6] and [8].

The surface $\Sigma_{(C: D)}$ given by the equation above is singular at 12 points and contains 32 lines. Sixteen of these lines are common to all the surfaces in the pencil. More precisely, we have the following result.

Theorem 3.8 (see $[6,8]$ ). If $C D \neq 0$, then $\Sigma_{(C: D)}$ has ordinary double points at the four poles $P_{0}, \ldots, P_{3}(=(1: 0: 0: 0)$, etc. $)$ and at the eight points $( \pm 1: \pm 1: \pm 1: \pm 1)$ forming the vertices of a cube, and no other singularities. A vertex is said to be even or odd according to whether the number of coordinates equal to -1 is even or odd. The 16 lines $L_{1}, \ldots, L_{16}$ that are the edges and main diagonals of the cube lie in $\Sigma_{(C: D)}$ : each passes through exactly one pole, one even point and one odd point.

The configuration of lines and points is the well-known Reye configuration (see also, in this context, $[13, \S \S 6-7])$.

We can resolve the singularities of $\Sigma_{(C: D)}$ by blowing up the nodes. Let $\beta: \tilde{\Sigma}_{(C: D)} \rightarrow$ $\Sigma_{(C: D)}$ be this blow-up and let $\tilde{L}_{j}$ be the proper transform of $L_{j}$ in $\tilde{\Sigma}_{(C: D)}$. Denote by $C_{i}^{0}, C_{i}^{+}$and $C_{i}^{-}$the exceptional curve in $\tilde{\Sigma}_{(C: D)}$ coming from the $i$ th pole, even and odd vertex, respectively.

Lemma 3.9. $\tilde{\Sigma}_{(C: D)}$ is a smooth $K 3$ surface.

Proof. $K_{\Sigma_{(C: D)}}=0$ by adjunction, and blowing up nodes does not change this.

The 16 rational curves $\tilde{L}_{j}$ are smooth and disjoint, so we can apply Nikulin's construction from [14] as before, obtaining

$$
A_{(C: D)} \stackrel{\sigma}{\longleftarrow} \tilde{A}_{(C: D)} \stackrel{f}{\rightarrow} \tilde{\Sigma}_{(C: D)} \stackrel{\beta}{\rightarrow} \Sigma_{(C: D)}
$$

where $f$ is a double cover branched along $\tilde{L}_{j}$ only, $\sigma$ is the blow-down of the 16 rational curves $f^{-1}\left(\tilde{L}_{j}\right)$, and $A_{(C: D)}$ is (after choosing an origin) an abelian surface. Furthermore, $\tilde{\Sigma}_{(C: D)}=\widehat{\mathrm{Km}} A_{(C: D)}$ and $f$ is the Kummer map.

* Greek $\delta \epsilon \sigma \mu o ́ s$, a band or tie, from $\delta \epsilon \epsilon \omega$, to bind. 
Theorem 3.10. $A_{(C: D)} \cong E \times E$, where $E$ is the elliptic curve whose $j$-invariant is $j(C / D)=2^{8}\left(C^{2}-C D+D^{2}\right)^{3} / C^{2} D^{2}(C-D)^{2}$.

Proof. Consider the curves $\tilde{E}_{i}^{0}=f^{-1}\left(\tilde{C}_{i}^{0}\right)$, etc. Each of these is an elliptic curve since $f: \tilde{E}_{i}^{0} \rightarrow \tilde{C}_{i}^{0}$ is a double cover branched at the four points corresponding to the four lines $L_{j}$ passing through $P_{i}$, and similarly for the odd and even vertices. These 12 elliptic curves are disjoint in $\tilde{A}_{(C: D)}$ but their images $E_{i}^{0}, E_{i}^{ \pm}$in $A_{(C: D)}$ have intersection numbers $E_{i}^{0} E_{j}^{0}=0, E_{i}^{0} E_{j}^{+}=E_{j}^{+} E_{k}^{-}=E_{k}^{-} E_{i}^{0}=1$. Indeed, $E_{i}^{0}, E_{j}^{+}$and $E_{k}^{-}$are concurrent.

There is an exact sequence of abelian varieties

$$
0 \longrightarrow E_{0}^{0} \longrightarrow A_{(C: D)} \longrightarrow E_{0}^{0 *} \longrightarrow 0,
$$

where $E_{0}^{0 *}$ is an elliptic curve. Since $E_{0}^{0} E_{j}^{+}=1$ it follows that $E_{j}^{+} \cong E_{0}^{0 *}$, and similarly $E_{k}^{-} \cong E_{0}^{0 *}$ for all $j, k$. Similarly, $E_{i}^{0} \cong E_{0}^{+*} \cong E_{0}^{-}$for all $i$, so all the elliptic curves $E_{i}^{0}, E_{j}^{+}, E_{k}^{-}, E_{i}^{0 *}, E_{j}^{+*}, E_{k}^{-*}$ are isomorphic to one another. Moreover, $E_{0}^{+}$defines a section of $A_{(C: D)} \rightarrow E_{0}^{0 *}$, so $A_{(C: D)} \cong E_{0}^{0} \times E_{0}^{+} \cong E \times E$.

To calculate the $j$-invariant of $E$ we need to know the cross-ratio of the four branch points of $f: \tilde{E}_{0}^{0} \rightarrow \tilde{C}_{0}^{0}$. We use $z_{1}, z_{2}$ and $z_{3}$ as affine coordinates in the affine piece $z_{0}=1$ of $\mathbb{P}^{3}$, so that $P_{0}$ is the origin of $\mathbb{A}^{3}$ and $\Sigma_{(C: D)}$ has the affine equation

$$
C\left(1-z_{3}^{2}\right)\left(z_{1}^{2}-z_{2}^{2}\right)+D\left(1-z_{2}^{2}\right)\left(z_{3}^{2}-z_{1}^{2}\right)=0 .
$$

The tangent cone to $\Sigma_{(C: D)}$ at $(0,0,0)$ has the equation

$$
C\left(z_{1}^{2}-z_{2}^{2}\right)+D\left(z_{3}^{2}-z_{1}^{2}\right)=0
$$

and this can also be thought of as the equation of $\tilde{C}_{0}^{0}$ in the $\mathbb{P}^{2}$ which is the set of lines in $\mathbb{A}^{3}$ through the origin. The branch points are given by the lines joining $P_{0}$ to the even points, which are $\left(1: \pm 1: \pm 1\right.$ ) (the vertices of a square). In the affine piece of $\mathbb{P}^{2}$ given by $z_{1}=1$, these points are simply $( \pm 1, \pm 1)$, and $\tilde{C}_{0}^{0}$ has the affine equation

$$
C\left(1-z_{2}^{2}\right)+D\left(z_{3}^{2}-1\right)=0 .
$$

If we take the isomorphism $\tilde{C}_{0}^{0} \rightarrow \mathbb{P}^{1}$ given by projection from $(-1,1)$, we find that the four points are mapped to $0,1, \infty$ and $C / D$.

In fact, the elliptic curves $E_{i}^{0}, E_{j}^{+}$and $E_{k}^{-}$are isomorphic for reasons of projective geometry, because there is an action of $\mathbb{S}_{6}$ that preserves $\Sigma_{(C: D)}$ and permutes the three tetrahedra parametrized by poles, odd vertices and even vertices.

This is case (IV) (the diagonal case) of $[2, \S 5]$. The $(1,3)$-polarization is given by $\mathcal{O}(E \times\{0\}+\{0\} \times E+\Delta)$, where $\Delta$ is the diagonal. The result has a long history (compare [7, p. 313], where another proof of part of this appears, or indeed [8]).

Acknowledgements. The original impetus for this work came from discussions between G.K.S. and I.N. while G.K.S. was visiting Mexico with support from the EU International Scientific Cooperation Programme Contract no. CI1*-CT93-0031. Most of 
the work on this paper was done during the visit of I.N. to Bath, supported by EPSRC grant no. GR/L27534, and Hannover in the autumn of 1996. I.N. was also supported by Conacyt project 0325P-E 'Moduli of Polarized Abelian Varieties'. K.H. and G.K.S. also acknowledge support from the EU HCM network AGE.

\section{References}

1. W. BARTh AND I. NiETO, Abelian surfaces of type $(1,3)$ and quartic surfaces with 16 skew lines, J. Alg. Geom. 3 (1994), 173-222.

2. T. Bauer, Projective images of Kummer surfaces, Math. Ann. 299 (1994), 155-170.

3. V. Gritsenko AND K. Hulek, Minimal Siegel modular threefolds, Math. Proc. Camb. Phil. Soc. 123 (1998), 461-485.

4. K. Hulek and S. Weintraub, Bielliptic abelian surfaces, Math. Ann. 283 (1989), 411429.

5. K. Hulek, I. Nieto and G. K. Sankaran, Degenerations of abelian surfaces of type $(1,3)$ and Kummer surfaces, in Algebraic Geometry: Hirzebruch 70 (ed. P. Pragacz, M. Szurek and J. Wiśniewski) (AMS Contemporary Mathematics, 1999).

6. R. W. H. T. Hudson, Kummer's quartic surface (Cambridge University Press, 1906; reissued 1990).

7. B. Hunt, The geometry of some special arithmetic quotients, Lecture Notes in Mathematics, no. 1637 (Springer, Berlin, 1996).

8. C. M. JESSOP, Quartic surfaces with singular points (Cambridge University Press, 1916).

9. H. LANGE AND CH. BiRKenhaKe, Complex abelian varieties (Springer, Berlin, 1992).

10. D. MUMFORD, On the equations defining abelian varieties, I, Inv. Math. 1 (1966), 287-354.

11. I. NARUKI, On smooth quartic embedding of Kummer surfaces, Proc. Jap. Acad. A67 (1991), 223-225.

12. I. NIETO, Invariante Quartiken unter der Heisenberg Gruppe $T, \mathrm{PhD}$ thesis, University of Erlangen, 1989.

13. I. NiETO, Examples of abelian surfaces with polarization type $(1,3)$, in Algebraic geometry and singularities (ed. C. Lopez and N. Macarro), Progress in Mathematics, vol. 134, pp. 319-337 (Birkhauser, Basel, 1996).

14. V. Nikulin, On Kummer surfaces, Math. USSR. Izv. 9 (1975), 261-275. 